\title{
Compression of klockmannite, CuSe
}

\author{
Suhithi M. Peiris ${ }^{\mathrm{a})}$ \\ Department of Chemistry, University of Chicago, Chicago, Illinois 60637 \\ Tania T. Pearson \\ Department of Chemistry, University of Michigan, Ann Arbor, Michigan 48109 \\ Dion L. Heinz \\ Department of Geophysical Sciences and James Franck Institute, University of Chicago, Chicago, \\ Illinois 60637
}

(Received 20 August 1997; accepted 1 April 1998)

\begin{abstract}
Copper selenide (CuSe) was compressed in a diamond anvil cell at room temperature up to a pressure of $52 \mathrm{GPa}$ and studied using energy dispersive x-ray diffraction and Raman spectroscopy. $\mathrm{CuSe}$ is nearly isostructural with copper sulfide $(\mathrm{CuS})$, and a previous study indicates that copper sulfide undergoes reversible pressure-induced amorphization at $18 \mathrm{GPa}$. The intensity of the x-ray diffraction peaks for $\mathrm{CuSe}$ decrease slowly, however, they never completely disappear up to a pressure of $52 \mathrm{GPa}$. The third-order Birch-Murnaghan equation of state fit to the data yields $K_{0}$ $=96.9 \pm 5.3 \mathrm{GPa}$ and $K_{0}^{\prime}=4.1 \pm 0.5$. Vinet's universal equation of state yields essentially identical parameters. Raman spectroscopy demonstrates that upon compression, the $\mathrm{S}-\mathrm{S}$ bond in $\mathrm{CuS}$ compresses differently than the $\mathrm{Se}-\mathrm{Se}$ bond in $\mathrm{CuSe}$, possibly accounting for the different high pressure behavior of these two very similar compounds. (c) 1998 American Institute of Physics.
\end{abstract} [S0021-9606(98)01926-6]

\section{INTRODUCTION}

$\mathrm{CuSe}$ is a slate-gray mineral that is commonly referred to as klockmannite. Klockmannite is similar to covellite $(\mathrm{CuS})$ in structure and composition. Extensive studies done by Earley and Berry established that klockmannite is isostructural with covellite. ${ }^{1,2}$ Both $\mathrm{CuS}$ and CuSe have a hexagonal crystal structure with trigonal-planar $\mathrm{CuX}_{3}$ units surrounded by tetrahedral $\mathrm{CuX}_{4}$ units. ${ }^{3,4}$ Therefore, the crystal is composed of layers of $\mathrm{CuX}_{4}-\mathrm{CuX}_{3}-\mathrm{CuX}_{4}$, which are held together by covalent bonding between $\mathrm{X}$ atoms from each layer. ${ }^{5}$ The only difference between $\mathrm{CuSe}$ and $\mathrm{CuS}$ is that CuSe has a hexagonal superstructure of $13 \mathrm{CuS}$-type subshells which is caused by inexact positioning of certain copper atoms in the trigonal-planar layer.

It has been reported that $\mathrm{CuSe}$ undergoes two phase transitions with increased temperature. The first phase transition occurs at $323 \mathrm{~K}$ from the hexagonal to an orthorhombic symmetry. Further increase in temperature leads in a transition to another hexagonal structure at $393 \mathrm{~K}$, where no superlattice reflections are observed. ${ }^{6}$ Isostructural $\mathrm{CuS}$ shows pressureinduced amorphization under high pressure, at $18 \mathrm{GPa}^{7}$ Thus, we wanted to observe the effect of compression on the CuSe lattice.

We report here the study of CuSe at high pressure with $\mathrm{x}$-ray diffraction and Raman spectroscopy using a diamond anvil cell. While increasing the pressure, the intensity of the x-ray diffraction patterns for CuSe decrease, but do not completely disappear up to $52 \mathrm{GPa}$. The diffraction data for $\mathrm{CuSe}$ were fit with Birch-Murnaghan and Vinet's universal equa-

\footnotetext{
a)Present address: Code 6110, Naval Research Lab, Washington, D.C. 20375 .
}

tions of state to obtain zero-pressure isothermal bulk moduli and pressure derivatives for CuSe. The difference in the Raman spectra of $\mathrm{CuS}$ and $\mathrm{CuSe}$ at high pressure, is evidence that the compression mechanisms of these two compounds are not identical.

\section{EXPERIMENT}

CuSe samples (99.5\%, Johnson Matthey Electronics, lot\# D25A22) were loaded into a Mao-Bell ${ }^{8}$ type diamond anvil cell at ambient temperature. The culets of the diamonds were about $250 \mu \mathrm{m}$ in diameter. Gaskets made of $127 \mu \mathrm{m}$ thick Inconel, were drilled with holes $150 \mu \mathrm{m}$ in diameter. After the gasket and hole were preindented to about $90 \mu \mathrm{m}$ in thickness and $80 \mu \mathrm{m}$ in diameter, a sample of crushed $\mathrm{CuSe}$ was loaded inside the gasket hole. Each sample was loaded in air with a few specs of gold ( $\mathrm{Au}$ ) powder mixed in as an internal pressure standard.

Synchrotron x-ray diffraction patterns were obtained with an energy dispersive Ge detector at beamline X17C of the National Synchrotron Light Source (NSLS) at Brookhaven National Laboratory. The detector was calibrated using the $K_{\alpha}$ lines of $\mathrm{Cu}, \mathrm{Rb}, \mathrm{Mo}, \mathrm{Tb}, \mathrm{Ba}$, and $\mathrm{Ag}$. $\mathrm{X}$-ray spectra were collected for 5-18 min with the detector at a $2 \theta$ angle of $15^{\circ}$ from the incident beam. With the detector at this angle, a value of $E-d$ (derived from $E=h \nu$ and Bragg's law $\lambda=2 d \sin \theta$ ) is $47.54684 \mathrm{keV} \AA$. The energy $(E)$ of each of the diffraction peaks in each spectrum was estimated by fitting a Gaussian curve to each peak. The 6-20 CuSe diffraction peaks were indexed according to the Joint Committee on Powder Diffraction Standards (JCPDS) card numbers 6-0427 and 20-1020. The unit cell volume was calculated using the average cell parameters of a hexagonal 


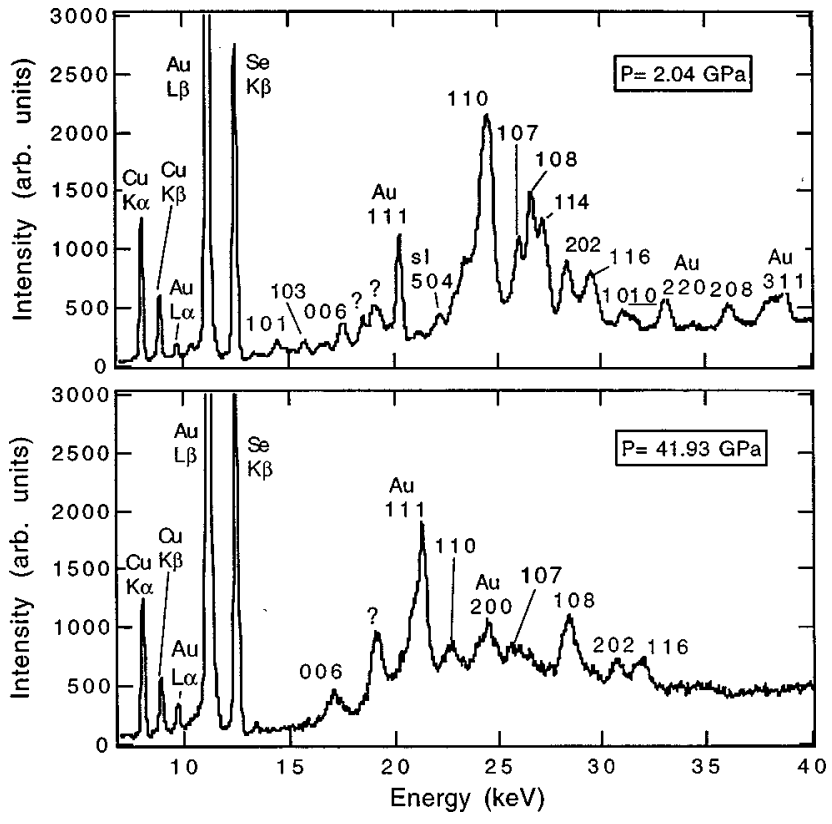

FIG. 1. X-ray diffraction spectra of CuSe at two different pressures. Both spectra are scaled to the intensity of the $\mathrm{Cu} K_{\alpha}$ peak.

crystal structure. Pressures were determined by calculating the gold unit cell volume from gold diffraction peaks and using a standard equation of state for gold. ${ }^{9}$

Raman spectra was obtained for two samples of $\mathrm{CuSe}$ at the Argonne National Laboratory up to a pressure of $50 \mathrm{GPa}$. The Raman system was in a nearly backscattered geometry with an incident angle of about $30^{\circ}$. Two separate lenses were used to focus the incident and scattered beams. Spectra was obtained using the $476.2 \mathrm{~nm}$ excitation of a $\mathrm{Kr}$ ion laser, a JY T64000 triple spectrometer, and a Princeton Instruments CCD array. Both samples were loaded with a methanol:ethanol:water (16:4:1) mixture as a pressure medium. Pressure was determined by using the in situ ruby fluorescence method ${ }^{10}$ with Raman spectroscopy.

\section{RESULTS}

X-ray diffraction patterns obtained at $2.04 \mathrm{GPa}$ and at $41.93 \mathrm{GPa}$, are shown in Fig. 1. All the spectra included many fluorescence lines of $\mathrm{Au}, \mathrm{Cu}$, and $\mathrm{Se}$. In this figure, we have used the $\mathrm{Cu} K_{\alpha}$ fluorescence line to scale the intensity of the two spectra presented. This scaling ensures that the compared intensities result from the same quantity of $\mathrm{CuSe}$, eliminating any effect on intensity due to sample thinning during compression, and allowing a real comparison of intensity of the diffracted lines. The spectrum at 2.04 GPa has more peaks than the spectrum at $41.93 \mathrm{GPa}$. As pressure is increased, the 101, 103, 110, 1010, and $208 \mathrm{CuSe}$ diffraction peaks lose intensity up to $52 \mathrm{GPa}$, while the peaks 006,107 , 108, 202, and 116 maintain almost consistent intensity. In Fig. 1, the 110 peak shows the most significant decrease in intensity; at $41.93 \mathrm{GPa}$ it is only $30 \%$ of the intensity at 2.04 GPa. Upon decompression of the sample, all but the 107 and $1010 \mathrm{CuSe}$ diffraction peaks regain intensity. The x-ray data that were obtained have been published elsewhere. ${ }^{15}$
There are three peaks (marked with "?'”) on the spectra presented in Fig. 1. We were unable to index these peaks to any $\mathrm{CuSe}$ lattice or superlattice lines, and we believe that these peaks are possibly due to an impurity in the commercially obtained samples. The initial lot of CuSe powder sent was more contaminated or was a different composition of $\mathrm{CuSe}$ than stoichiometric 1:1. All the data presented here was obtained from a second batch of CuSe sent by Johnson Matthey, and electron microprobe analysis was performed to ensure correct stoichiometry, and minimum impurities.

Elastic parameters were calculated by fitting Birch-Murnaghan $^{11}$ and Vinet's ${ }^{12}$ universal equations of state to the diffraction data. The second order BirchMurnaghan (BM) equation of state yields a bulk modulus of $98.48 \pm 1.59 \mathrm{GPa}$, where $K_{0}^{\prime}$ is held at a constant of 4.0. The third order BM equation of state, where $K_{0}^{\prime}$ is allowed to vary gives a statistically better fit, yielding a bulk modulus of 96.9 $\pm 5.3 \mathrm{GPa}$ and a pressure derivative of $4.14 \pm 0.47$. Vinet's universal equation of state yields a bulk modulus of $97.81 \pm 5.67 \mathrm{GPa}$, with a pressure derivative of $4.24 \pm 0.55$.

The Raman spectra obtained for $\mathrm{CuSe}$ at ambient pressure concurs with Iishi. ${ }^{13}$ However, the peak at $17 \mathrm{~cm}^{-1}$ was not observed because the detector was saturated by the Raleigh peak below $35 \mathrm{~cm}^{-1}$. Spectra obtained at $2.37 \mathrm{GPa}$, 16.63 GPa, and 39.67 GPa are presented in Fig. 3, and they contain many krypton laser plasma lines and Ar calibration lines. The peaks due to Raman scattering by $\mathrm{CuSe}$ are labeled in the $2.37 \mathrm{GPa}$ spectrum. The peak at $263 \mathrm{~cm}^{-1}$ (labeled as 270 in Fig. 3) at $2.37 \mathrm{GPa}$, moves to a slightly higher frequency upon compression. At $16.63 \mathrm{GPa}$ the peak splits in two, and starts to lose intensity. By $39.67 \mathrm{GPa}$, the double peak is still visible, but with very low intensity. The peak at $44 \mathrm{~cm}^{-1}$ overlaps with the $\mathrm{Kr}$ plasma peak and appears at $55 \mathrm{~cm}^{-1}$ as a very intense peak. Upon compression, the intensity of this peak increases. The small peak at $207 \mathrm{~cm}^{-1}$ is also overshadowed by the $\mathrm{Kr}$ peak at $215 \mathrm{~cm}^{-1}$.

\section{DISCUSSION}

Although $\mathrm{CuSe}$ and $\mathrm{CuS}$ are similar in composition and structure, our studies have shown that as pressure increases, each compound behaves in a different manner. The bulk modulus for $\mathrm{CuSe}$ is $96.92 \pm 5.31 \mathrm{GPa}$ with a pressure derivative of $4.14 \pm 0.47$. In comparison, the bulk modulus of $\mathrm{CuS}$ is $89 \pm 10 \mathrm{GPa}$ with a pressure derivative of $-2 \pm 2{ }^{7}$ The higher the bulk modulus, the less compressible the compound. Therefore, $\mathrm{CuSe}$ is a more rigid structure than $\mathrm{CuS}$, possibly due to the higher covalency of $\mathrm{Cu}-\mathrm{Se}$ bonds in comparison with $\mathrm{Cu}-\mathrm{S}$ bonds. The compression curve of $\mathrm{CuSe}$ (Fig. 2) is typical of many minerals, where $K_{0}^{\prime}$ has a value close to 4 . However, $\mathrm{CuS}$ has a negative $K_{0}^{\prime}$ value, which indicates that it is thermodynamically unstable at high pressures, and compression to $18 \mathrm{GPa}$ results in amorphization of $\mathrm{CuS}$. In addition, though both $\mathrm{CuSe}$ and $\mathrm{CuS}$ have identical symmetry, CuSe also has a superstructure due to inexact positioning of copper atoms in the trigonal-planar layer. These facts, together with the larger size of the Se atoms in comparison to $\mathrm{S}$ atoms, help us understand the differences in the compression of these two compounds. 


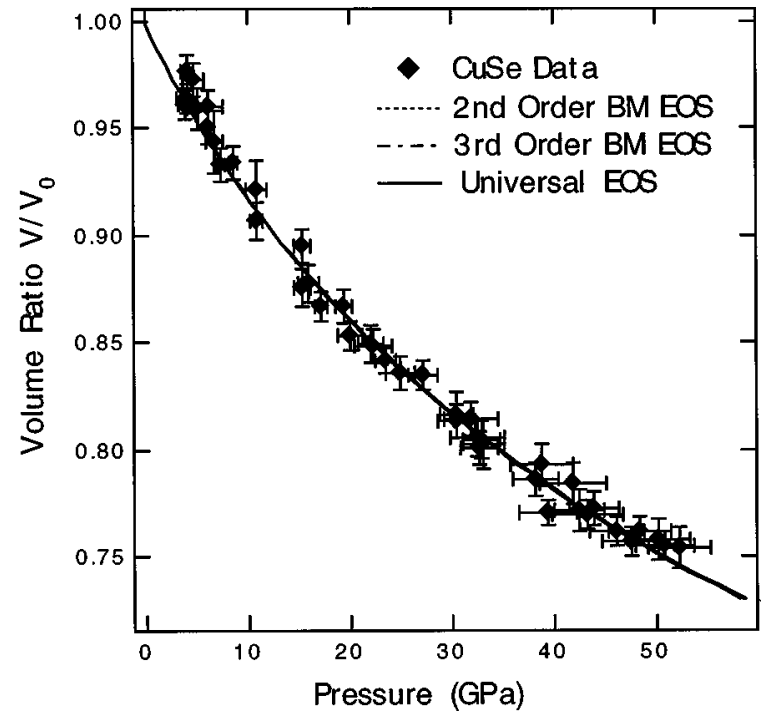

FIG. 2. Pressure vs $V / V_{0}$, where $V_{0}=231.059 \AA^{3}$, using CuSe compression data. Birch-Murnaghan and Vinet's universal equations of state fitted to the data are the dotted and solid lines.

Upon compression, diffraction experiments show a loss of intensity of certain CuSe peaks. The decrease in intensity due to the thinness of the sample is accounted for by scaling the spectra in Fig. 1 according to the $\mathrm{Cu} K_{\alpha}$ peak. However, up to $52 \mathrm{GPa}$, there are $\mathrm{CuSe}$ peaks that remain in the $\mathrm{x}$-ray diffraction spectrum that can be indexed to the ambient pressure $\mathrm{CuSe}$ structure. Therefore, CuSe does not completely amorphize to $52 \mathrm{GPa}$, unlike CuS. Instead the loss of intensity of certain peaks may also imply some preferred orientations within the lattice or a minor phase change.

Pressure-induced amorphization is considered to be a transition from a stable to an unstable phase. $\mathrm{CuS}$ undergoes a transition to an orthorhombic phase at a temperature of 55 $\mathrm{K} .{ }^{14}$ Since the effect of low temperature is thermodynamically similar to increased pressure, the mechanism by which $\mathrm{CuS}$ goes amorphous has been discussed as a kinetically hindered transition to the low temperature orthorhombic phase. ${ }^{7}$ $\mathrm{CuSe}$ undergoes a similar transition to an orthorhombic phase, but at $323 \mathrm{~K}$, a temperature higher than room temperature. ${ }^{6}$ This transition then is due to an expansion effect as opposed to the effect of compression. Thus, if the loss of CuSe diffraction intensity does result in amorphization at a pressure higher than $52 \mathrm{GPa}$, the mechanism for amorphization would be different to that of the amorphization of $\mathrm{CuS}$.

According to Iishi ${ }^{13}$ the peak labeled 270 (in Fig. 3) in the Raman spectra of CuSe is assigned the $\mathrm{Se}-\mathrm{Se}$ stretch. Upon compression, this peak splits into two, but retains a position close to the ambient pressure position of $263 \mathrm{~cm}^{-1}$. The peak splitting indicates that there are two different $\mathrm{Se}-\mathrm{Se}$ bond lengths at high pressure. This observation suggests that the structure is undergoing a minor phase transformation to another crystalline phase. However, because this

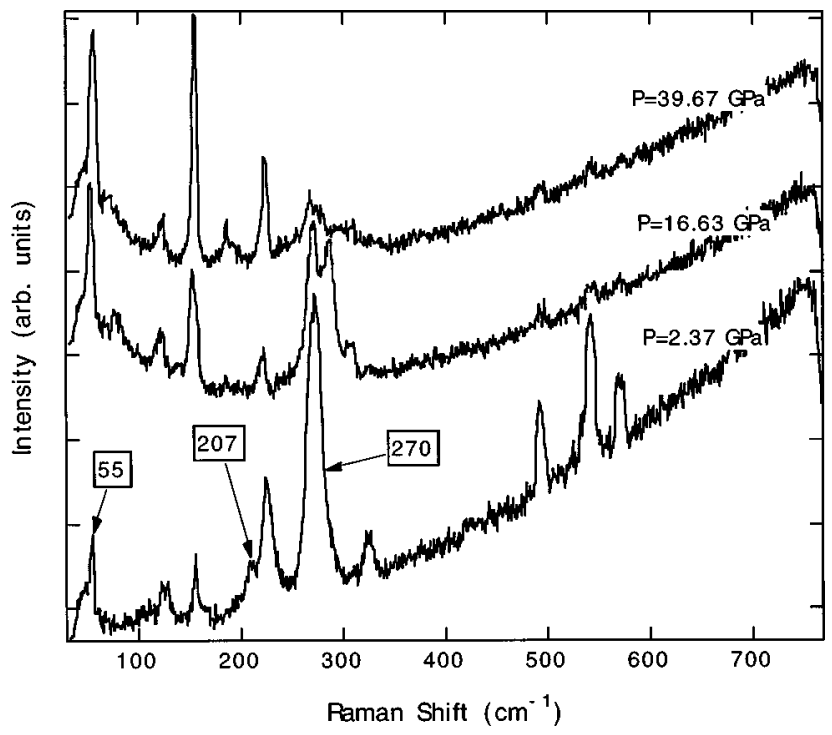

FIG. 3. Raman spectra of CuSe. The peaks due to Raman scattering from $\mathrm{CuSe}$ are labeled according to their position at $2.37 \mathrm{GPa}$.

peak also loses intensity by $39 \mathrm{GPa}$, implying the breakdown of this $\mathrm{Se}-\mathrm{Se}$ bond vibration, the amorphization of $\mathrm{CuSe}$ at a pressure higher than $52 \mathrm{GPa}$ is also a relevant possibility.

\section{CONCLUSION}

We have performed $\mathrm{x}$-ray diffraction experiments on CuSe while compressing the sample in a diamond anvil cell. As pressure increases, the intensity of a number of $\mathrm{CuSe}$ peaks decrease. The equations of states calculated for $\mathrm{CuSe}$ yielded an average bulk modulus of $97.25 \pm 5.49 \mathrm{GPa}$ with an average pressure derivative of $4.19 \pm 0.51$. Raman spectroscopy of CuSe indicates that the Se-Se bond splits in two suggesting different $\mathrm{Se}-\mathrm{Se}$ bond lengths. These observations can be explained by either pressure-induced amorphization or a crystalline-crystalline phase transition in CuSe at a pressure higher than $52 \mathrm{GPa}$.

${ }^{1}$ L. G. Berry, Am. Mineral. 39, 504 (1949).

${ }^{2}$ J. W. Earley, Am. Mineral. 34, 435 (1954).

${ }^{3}$ H. T. Evans Jr. and J. A. Konnert, Am. Mineral. 61, 996 (1976).

${ }^{4}$ W. Liang and M.-H. Whangbo, Solid State Commun. 85, 405 (1993).

${ }^{5}$ H. J. Gotsis, A. C. Barnes, and P. Strange, J. Phys.: Condens. Matter 4, 10461 (1992).

${ }^{6}$ H. Nozaki et al., Mater. Res. Bull. 29, 203 (1994).

${ }^{7}$ S. M. Peiris, J. S. Sweeney, A. Campbell, and D. L. Heinz, J. Chem. Phys. 104, 11 (1996).

${ }^{8}$ H. K. Mao, P. M. Bell, K. J Dunn, R. M. Chrenko, and R. C. Devries, Rev. Sci. Instrum. 50, 1002 (1979).

${ }^{9}$ D. L. Heinz and R. Jeanloz, J. Appl. Phys. 55, 885 (1984).

${ }^{10}$ H. K. Mao, P. M. Bell, J. W. Shaner, and D. J. Steinberg, J. Appl. Phys. 49, 3276 (1978)

${ }^{11}$ F. Birch, J. Geophys. Res. 83, 3543 (1978).

${ }^{12}$ P. Vinet, J. Ferrante, J. H. Rose, and J. R. Smith, J. Geophys. Res. 92, 9319 (1987)

${ }^{13}$ M. Iishi, K. Shibata, and H. Nozaki, J. Solid State Chem. 105, 504 (1993).

${ }^{14}$ H. Fjellvag, F. Gronvold, S. Stolen, A. F. Andersen, R. Muller-Kafer, and A. Simon, Z. Kristallogr. 184, 111 (1988).

${ }^{15}$ S. M. Peiris, Ph.D. dissertation, University of Chicago, 1996. 